\title{
Design, 3D printing and validation of a novel low-cost high-capacity sitting-drop bridge for protein crystallization
}

\author{
Sandeep K. Talapatra, Matthew R. Penny, Stephen T. Hilton and Frank \\ Kozielski
}

J. Appl. Cryst. (2019). 52, 171-174

\section{IUCr Journals CRYSTALLOGRAPHY JOURNALS ONLINE}

Copyright (C) International Union of Crystallography

Author(s) of this paper may load this reprint on their own web site or institutional repository provided that this cover page is retained. Republication of this article or its storage in electronic databases other than as specified above is not permitted without prior permission in writing from the IUCr.

For further information see http://journals.iucr.org/services/authorrights.html 
JOURNAL OF

APPLIED

CRYSTALLOGRAPHY

ISSN 1600-5767

Received 30 August 2018

Accepted 11 December 2018

Edited by F. Meilleur, Oak Ridge National Laboratory, USA, and North Carolina State University, USA

₹ Current address: Discovery Biology, Discovery Sciences, IMED Biotech Unit, AstraZeneca, Alderley Park, UK.

Keywords: protein crystallization; sitting-drop crystallization; hanging-drop crystallization; vapour-diffusion crystallization; microbridges; 3D printing.

Supporting information: this article has supporting information at journals.iucr.org/j
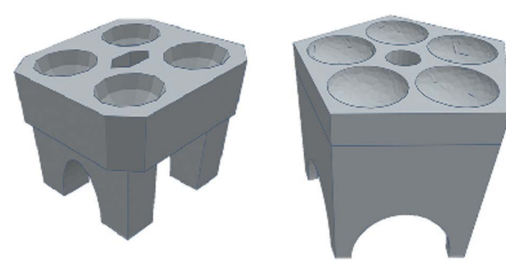

(C) 2019 International Union of Crystallography

\section{Design, 3D printing and validation of a novel low- cost high-capacity sitting-drop bridge for protein crystallization}

\author{
Sandeep K. Talapatra, ${ }^{*}$ Matthew R. Penny, Stephen T. Hilton and Frank Kozielski*
}

Department of Pharmaceutical and Biological Chemistry, School of Pharmacy, University College London, 29-39 Brunswick Square, London WC1N 1AX, UK. *Correspondence e-mail: s.talapatra@ucl.ac.uk, f.kozielski@ucl.ac.uk

Sitting-drop protein crystallization is not used as commonly as the hanging-drop method for crystal optimization owing to the limitations of commercially available sitting-drop bridges, particularly when they are used in conjunction with 24-well crystallization plates. The commercially available sitting-drop bridge, containing space for only a single drop, restricts their wider use. Proteins that preferentially crystallize under sitting-drop conditions therefore require more work, time and resources for their optimization. In response to these limitations, and using 3D printing, a new sitting-drop bridge has been designed and developed, where five crystallization drops can be placed simultaneously in each well of a 24-well crystallization plate. This significantly simplifies the process and increases the potential of sitting drops in crystal optimization, reducing costs and hence overcoming the limitations of current approaches.

\section{Introduction}

Protein crystallization using the vapour-diffusion method is the most commonly employed crystallization technique (McRee, 1993). Among several bottlenecks in protein crystallography, the optimization of protein crystals is one of the key factors in obtaining high-resolution protein structures (McRee, 1993; McRee \& David, 1999). The majority of proteins crystallize under both sitting- and hanging-drop vapour-diffusion experiments (Dessau \& Modis, 2011). However, a few proteins undergo nucleation and crystal formation preferentially or exclusively in sitting-drop conditions (Fresco et al., 1968; Rayment, 2002; Soundararajan et al., 2013; Kozielski et al., 1999). Common practice in these cases is the use of well established and commercially available 24-well crystallization plates such as Linbro plates, in conjunction with a one-position sitting-drop bridge. Hanging-drop crystallization experiments are set up using a siliconized coverslip, allowing the simultaneous preparation of multiple crystallization drops on the same coverslip. Ideally, in this case, up to five different drops, each having a final volume of up to $3 \mu \mathrm{l}$ under a single crystallization condition (or per crystallization well), can readily be prepared. Crystallization drops composed of larger volumes reduce the overall number of drops which can be placed on the same coverslip. Initial optimization typically involves the variation of drop volumes, protein concentration, crystallization buffer, additives etc. Therefore, the need to place more than a single drop per well is highly desired for quickly obtaining optimized high-quality and well diffracting crystals. However, current commercially available sitting-drop bridges based on the published design (Harlos, 1992; Hubálek et al., 2003) do not allow this flexibility and 
multiple numbers of wells or plates have to be set up to achieve the same throughput as obtained with the hangingdrop method.

In order to streamline the sitting-drop method and align its efficiency with the hanging-drop method, and on the basis of our experience in developing low-cost equipment using 3D printing (Tyson et al., 2015; Mohmmed et al., 2016), we have designed a five-position sitting-drop bridge to be used in conjunction with widely used and commercially available 24well Linbro crystallization plates. In addition, we have tested our newly designed bridge with a well characterized protein to demonstrate the effectiveness of the new five-position bridge.

\section{Concept and design}

The five-position sitting-drop bridge design we present here was originally conceptualized keeping in mind the presently available sitting-drop methods used in 24-well plates for protein crystallization and optimization. Our first design worked on the basis of a four-position sitting-drop bridge with a compact fit within the well [Fig. 1(a)]. Initially, to increase the capacity of the sitting-drop bridge we looked at two potential approaches. The first focus was on the design of a new crystallization plate rather than using the established 24well Linbro plate. The second approach was based on modifying the design of the sitting-drop bridge to have increased capacity, without undermining the subsequent processes to obtain high-quality crystals. Given the wide use of the 24-well plate and the established design and setup, we decided to pursue the second approach. Using 3D printing, we initially fabricated a four-position bridge, and we tested it with dengue virus RNA-dependent RNA polymerase serotype 3 (DENV3 $\mathrm{RdRp}$ ). This protein is well characterized to form crystals irrespective of the type of method used and it consistently diffracts to a high resolution (Noble et al., 2016). Although we obtained crystals using the four-position bridge, there were two underlying concerns we wanted to address further. Firstly, we wished to optimize the maximum space available in a single well without compromising the stability of the bridge. Secondly, we wanted to generate a better shape and contouring of the drops so that crystals were easier to observe and harvest under a normal light microscope similar to the one used for crystals grown in hanging drops.

Using 3D printing, we then designed a five-position sittingdrop bridge, which improved the fit within the individual well and further increased the number of crystallization drops that can be set up simultaneously. In addition, the shape of each position was modified in such a fashion that the observation of protein crystals under an optical microscope was improved and the process of 'fishing' for crystals using loops was significantly simplified by allowing this to be done at a more convenient angle.

\section{3D-printing details}

The sitting-drop bridges were designed using either Tinkercad or Autodesk 123D (Autodesk, San Rafael, California, USA) and exported as . STL files. 3D printing was carried out using a Formlabs (Somerville, Massachusetts, USA) Form 2 SLA 3D printer using Formlabs Clear Resin V4 with a layer height of $0.1 \mathrm{~mm}$. Care was taken to ensure that support attachment points were not located underneath wells. After printing, the sitting-drop bridges were immersed in propan-2-ol for $15 \mathrm{~min}$, dried and then cured using Formlabs Form Cure for $30 \mathrm{~min}$ at $333 \mathrm{~K}$.

Although the sitting-drop bridges were printed using clear resin, the layers of the $3 \mathrm{D}$ print hampered visualization of the crystals under the microscope owing to refraction of light underneath the well. To improve transparency, a thin layer of Clear Resin V4 was added to the inside surface of the wells (a)

(i)

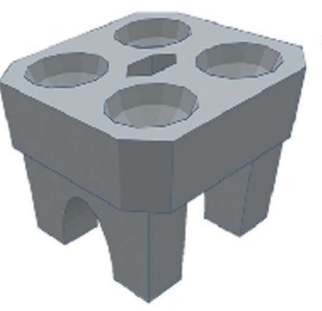

(b)

(i)

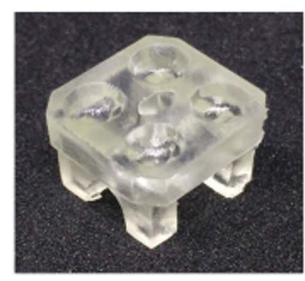

(ii)

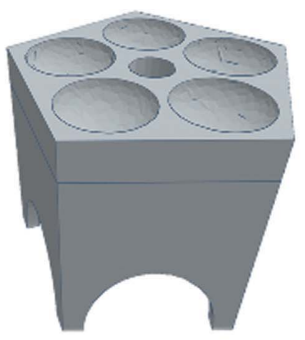

(ii)

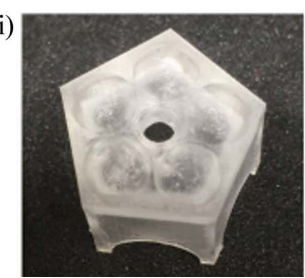

(d)
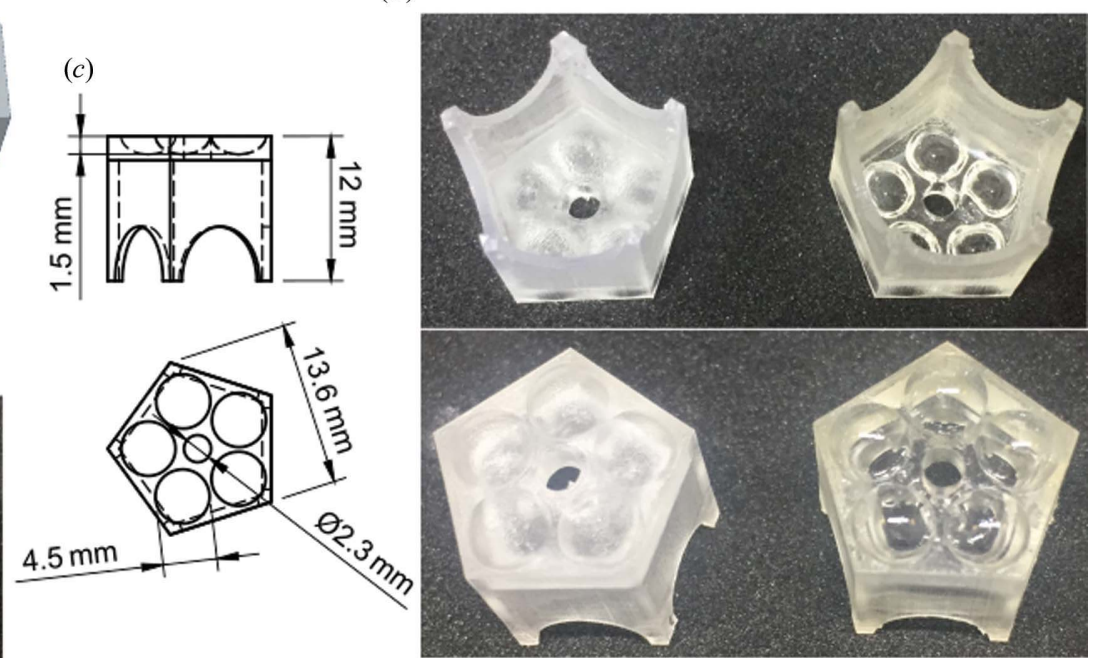

Figure 1

Conceptualization, design and structure statistics. (a) CAD image of (i) the initial four-position design and (ii) the optimized five-position design. (b) Photographs of (i) the initial four-position microbridge and (ii) the optimized five-position microbridge. (c) The dimensions of the optimized design. (d) Photographs showing the microbridge (left) before and (right) after treatment with additional resin to improve transparency. 
and the models were cured for a further $30 \mathrm{~min}$ at $333 \mathrm{~K}$. This procedure was also carried out for the surface directly underneath the wells (after detachment of support structures) and repeated twice until a satisfactory clarity was achieved.

Although we adapted our new five-position sitting-drop bridge to the widely used 24-well Linbro crystallization plate, our design can easily be tailored to fit other less frequently used crystallization plates. The cost of materials for fabrication is just USD 0.32 per microbridge, which includes the Formlabs Clear Resin and propan-2-ol. We are able to print up to 25 microbridges per printer at one time, taking a total of $5 \mathrm{~h}$ (including printing time, washing and finishing), which equates to less than $15 \mathrm{~min}$ per microbridge. Manual procedures for the whole process take less than 2 min per microbridge. The 3D printing file for our microbridge design is provided with this article.

\section{Application and future use}

4.1. Set up of crystallization trials and verification of volume and ratio of protein versus reservoir solution

To verify and validate the new five-position sitting-drop bridge that we designed, we carried out crystallization experiments in 24-well Linbro plates using both hanging-drop and sitting-drop experiments with the conventional one- position bridge and our new five-position bridge. Crystallization trials were set up using ratios of 1:1, 1:2, 2:1, 2:2 and 3:1 $\mu$ l of DENV3 RdRp and the reservoir solution (crystallization condition), respectively. For the one-position sittingdrop bridges, the standard 1:1 $\mu$ l condition was used. DENV3 RdRp crystals were obtained under all conditions, with sizes varying between 10 and $40 \mu \mathrm{m}$ [Fig. 2(a)].

\subsection{Comparison of crystal quality from hanging drops and from one-position and five-position sitting-drop bridges}

To compare the quality of the crystals obtained in the fiveposition sitting-drop bridge with the hanging drop or oneposition sitting-drop bridge, ten to 15 crystals from each experiment with sizes ranging between 10 and $40 \mu \mathrm{m}$ were harvested for diffraction measurements. The crystals were selected by simple visual examination under a light microscope.

We collected data sets and processed them with either $X D S$ (Kabsch, 2010) or iMOSFLM (Battye et al., 2011). Using stringent criteria for data-collecting statistics, we were able to compare the three different approaches to obtaining crystals. We initially selected data sets with $R_{\text {merge }}$ values lower than 0.12 . Next, we selected data sets which had an $I_{\text {mean }} / \Sigma I_{\text {mean }}$ of at least 2.0 in the outer shell and an overall completeness of at least $99 \%$. The average resolution of these data sets was $2.0 \AA$. (b) (i)

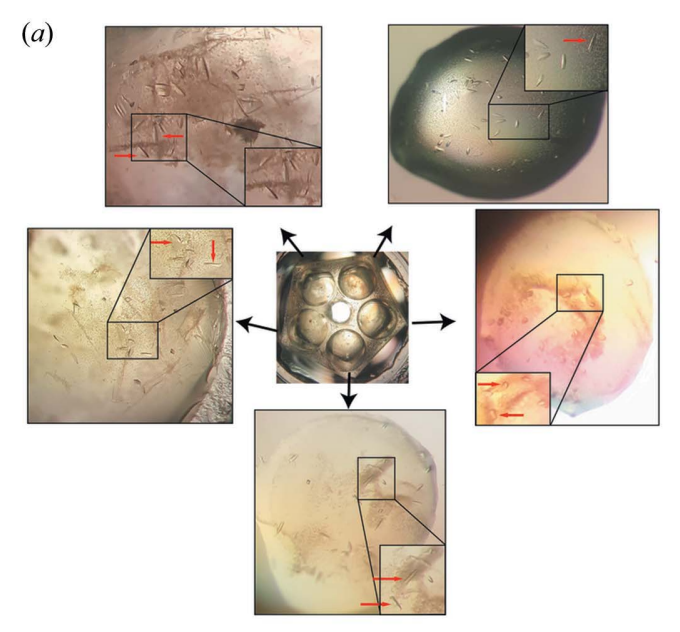

Figure 2

Crystallization experiments using the new five-position sitting-drop bridge. (a) RdRp crystals obtained in each individual position of the new fiveposition sitting-drop bridge. Red arrows point to DENV3 RdRp crystals used for diffraction measurements. (b) Crystals from individual drops were selected for diffraction measurements and crystal quality assessments. Shown here are comparisons of various collection statistics [panel (i) overall $R_{\text {merge }}$, (ii) resolution, (iii) $I_{\text {mean }} / \Sigma I_{\text {mean }}$ of the outer shell and (iv) completeness] of ten different crystals of DENV3 RdRp obtained using the hangingdrop method, a one-position sitting-drop bridge and our new five-position sitting-drop bridge. There is no significant difference in crystal quality.

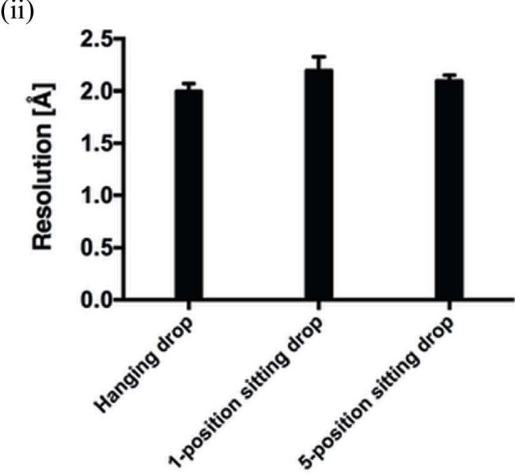

(iv)
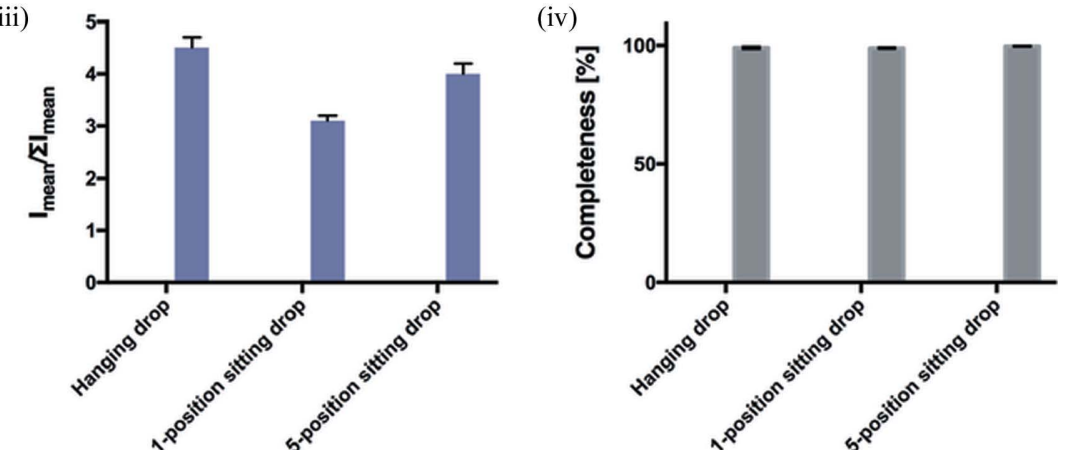


\section{short communications}

We were able to obtain at least ten good data sets for all three setups for comparison, as shown in Fig. 2(b), panels (i)-(iv). Therefore, we conclude that our novel five-position sittingdrop bridges do not affect the overall quality of the crystals or subsequent data-collection statistics.

\section{Acknowledgements}

We would like to thank Dr Michella Mazzon (UCL MRC Laboratory for Molecular Cell Biology) for providing the expression plasmid for DENV3 RdRp. We also thank Diamond Light Source for access to beamline I24 (MX17201) that contributed to the results presented here.

\section{References}

Battye, T. G. G., Kontogiannis, L., Johnson, O., Powell, H. R. \& Leslie, A. G. W. (2011). Acta Cryst. D67, 271-281.

Dessau, M. A. \& Modis, Y. (2011). J. Vis. Exp. 16, 47.
Fresco, J. R., Blake, R. D. \& Langridge, R. (1968). Nature, 220, 1285 1287.

Harlos, K. (1992). J. Appl. Cryst. 25, 536-538.

Hubálek, F., Binda, C., Li, M., Mattevi, A. \& Edmondson, D. E. (2003). Acta Cryst. D59, 1874-1876.

Kabsch, W. (2010). Acta Cryst. D66, 125-132.

Kozielski, F., De Bonis, S., Burmeister, W. P., Cohen-Addad, C. \& Wade, R. H. (1999). Structure, 7, 1407-1416.

McRee, D. E. (1993). Practical Protein Crystallography. San Diego: Academic Press.

McRee, D. E. \& David, P. R. (1999). Practical Protein Crystallography, 2nd ed. San Diego: Academic Press.

Mohmmed, S. A., Vianna, M. E., Penny, M. R., Hilton, S. T., Mordan, N. \& Knowles, J. C. (2016). Dent. Mater. 32, 1289-1300.

Noble, C. G., Lim, S. P., Arora, R., Yokokawa, F., Nilar, S., Seh, C. C., Wright, S. K., Benson, T. E., Smith, P. W. \& Shi, P. Y. (2016). J. Biol. Chem. 291, 8541-8548.

Rayment, I. (2002). Structure, 10, 147-151.

Soundararajan, M., Roos, A. K., Savitsky, P., Filippakopoulos, P., Kettenbach, A. N., Olsen, J. V., Gerber, S. A., Eswaran, J., Knapp, S. \& Elkins, J. M. (2013). Structure, 21, 986-996.

Tyson, A. L., Hilton, S. T. \& Andreae, L. C. (2015). Int. J. Pharm. 494, 651-656. 